\section{La estrategia de congelación, descongelación y maduración mejora la suavidad de los músculos del cuarto delantero de toretes sin castrar de diferentes fenotipos}

María Salud Rubio Lozano ${ }^{\text {a* }}$

Esmeralda Vanessa Pérez Buitrón ${ }^{\text {al }}$

Rubén Danilo Méndez Medinab

Adrián Chávez Gómez ${ }^{\mathrm{a}}$

Enrique Jesús Delgado Suárez ${ }^{\mathrm{c}}$

a Laboratorio de Ciencia de la Carne,

Secretaría de Producción Animal

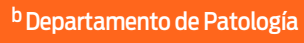

'Departamento de Medicina Preventiva y Salud Pública

Facultad de Medicina Veterinaria y Zootecnia, Universidad Nacional Autónoma de México, Av. Universidad 3000, Del. Coyoacán, Ciudad

Universitaria, México DF 04510

'Este artículo es el resultado de la investigación realizada por la segunda autora para obtener el grado de Maestra en Ciencias de la Salud y de la Producción Animal de la Universidad Nacional Autónoma de México.

*Autor para correspondencia: Tel: + 525558480514

Correo electónico: msalud@unam.mx

Recibido: 2014-04-22 Aceptado: 2014-09-24 Publicado: 2014-09-29

Información y declaraciones adicionales en la página 6

(a) Derechos de autor: María Salud Rubio Lozano et al. 2014

acceso abierto $\boldsymbol{O}$

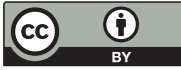

Distribuido bajo una Licencia Creative Commons

Atribución 4.0 Internacional (CC-BY 4.0)

\section{Resumen}

Se evaluó el efecto de la congelación seguida de la descongelación y maduración por 2, 9, 16 y 23 días en la fuerza de corte Warner-Bratzler (FC) en 11 músculos del cuarto delantero de toretes sin castrar de origen Brahman $(B R, n=10)$ y Charolais $(C H, n=10)$ seleccionados fenotípicamente. Splenius, infraspinatus, teres major, rhomboideus, y subscapularis de ambos fenotipos, y complexus de $\mathrm{CH}$ tuvieron valores típicos de carne suave (FC $\leq 40 \mathrm{~N}$ ) a los 9 días de maduración. La FC no mejoró después de los 9 días de maduración. En general, se observó que la congelación con una posterior descongelación y maduración de varios de los músculos estudiados mejoró significativamente la suavidad de la carne. Se requiere realizar otros estudios para optimizar esta estrategia de ablandamiento, así como para evaluar su posible impacto en otros atributos de la calidad de la carne.

Palabras clave: Calidad de la carne; Maduración; Músculos de la espaldilla de bovino; Fuerza de corte Warner-Bratzler; Machos jóvenes sin castrar.

\section{Introducción}

La maduración es una de las técnicas más usadas para mejorar la suavidad de la carne de res, la cual es el factor más importante en la calidad de la misma. Belew et al., 2003; Molina et al., 2005; Rhee et al., 2004; Von Seggern et al., 2005; Yadata et al., 2009 han investigado el proceso de ablandamiento de diversos músculos de bovino en diferentes periodos de maduración post-mortem. Sin embargo, la mayoría de los estudios se han hecho con machos castrados y hembras, a pesar de que los machos jóvenes sin castrar son la principal fuente de carne de bovino en muchos países (Mendez et al., 2009).

El escaso número de análisis con toretes (Chávez et al., 2012; Hildrum et al., 2009; Rubio et al., 2007; Simões et al., 2005; Vieira et al., 2009) ha mostrado que la carne de estos animales es dura. Los altos valores de FC reportados por estos autores indican que este tipo de carne es difícil de suavizar con la maduración. No obstante, Crouse y Koohmaraie (1990) han demostrado que la congelación antes 
de la maduración reduce la actividad de la calpastatina (Koohmaraie, 1990). En este sentido, Grayson et al., (2014), en su reciente estudio, señalan que la carne de res USDA Select, previamente congelada y descongelada antes de la maduración, se ablandó con mayor rapidez que aquella que no se había congelado. Por lo tanto, la estrategia de congelar-descongelar-madurar sería también un método efectivo para suavizar la carne de los toretes. Esto supondría una alternativa factible en algunos mercados con requerimientos específicos.

El objetivo de esta investigación es estimar el efecto de la congelación, descongelación y maduración en la suavidad de la carne de músculos del cuarto delantero de toretes producidos en México.

\section{Materiales y métodos}

Muestras de carne

Se estudiaron músculos de machos enteros de 18-24 meses de edad que se obtuvieron de dos rastros Tipo Inspección Federal (TIF) de México. En México se crían razas de los dos troncos genéticos (Bos taurus y Bos indicus), por lo que se decidió incluir en el análisis, al menos una de las razas con más uso de cada genotipo. Personal entrenado seleccionó fenotípicamente toretes Bos taurus (Charolais, $\mathrm{n}=10$ ) y Bos indicus (Brahman, $\mathrm{n}=10$ ) durante la estancia en los corrales de descanso del rastro. Los animales que no cumplían con las características fenotípicas de cada raza no fueron seleccionados para el estudio.

El estudio se subdividió en dos ensayos independientes, debido a la dificultad de encontrar un rastro donde ambos genotipos estuvieran disponibles. El ganado de cada uno de los fenotipos se originó en diferentes corrales de engorda y fue sacrificado en dos diferentes rastros. Sin embargo, se seleccionaron rastros y corrales de engorda con condiciones similares de manejo. A todos los animales se les alimentó con dietas de concentrado a base de sorgo, maíz, frijol de soya y pre-mezcla de vitaminas y minerales, en promedio por 100 días. Además, se criaron con la misma preparación de zilpaterol (Zilmax, Intervet México, S.A. de C.V.), añadiendo $0.006 \mathrm{~g} / \mathrm{kg}$ en la dieta durante 40 días antes del sacrificio (sin tiempo de retiro). Igualmente, la distancia desde cada corral de engorda al rastro fue de menos de $100 \mathrm{~km}$.

Las canales se sometieron a estimulación eléctrica (680 V, 0.3-0.5 A por 1015 s) entre 1 y 3 minutos después del desangrado, siguiendo las prácticas comunes de matanza en los rastros TIF de México. Después se refrigeraron a $0{ }^{\circ} \mathrm{C}$ por 24 horas. Entonces, personal entrenado separó un total de 11 músculos (biceps brachii, brachialis, complexus, splenius, infraspinatus, teres major, rhomboideus, subscapularis, supraspinatus, triceps brachii [cabeza larga], y triceps brachii [cabeza lateral]) del cuarto delantero. Cuatro cortes de $2.5 \mathrm{~cm}$ de ancho y $6 \mathrm{~cm}$ de diámetro fueron obtenidos de cada músculo de cada canal. Los cortes se obtuvieron sucesivamente desde la parte anterior de cada músculo, con ello se aseguró que todos fueran tomados del mismo sitio en cada una de las canales. Por último, las muestras se etiquetaron, empacaron al vacío y transportaron al Laboratorio de Ciencia de la Carne de la Facultad de Medicina Veterinaria y Zootecnia de la Universidad Nacional Autónoma de México, en la Ciudad de México. Los paquetes se trasladaron por carretera o avión en hieleras aisladas con geles refrigerantes y llega- 
ron al laboratorio después de 8-10 horas. Por lo tanto, los cortes tuvieron un total de maduración post-mortem de $32-34$ horas antes de ser congelados $\left(-30^{\circ} \mathrm{C}\right)$ por 7 días. Después de este tiempo, las muestras se descongelaron 24-30 horas a $2-4{ }^{\circ} \mathrm{C}$ y se asignaron aleatoriamente a uno de los siguientes tiempos de maduración: 0, 7, 14 o 21 días. Los cortes asignados a cero días de maduración se midieron de inmediato para determinar WBSF. Los tres cortes restantes fueron almacenados a $2-4{ }^{\circ} \mathrm{C}$ por los periodos de maduración designados. Como se mencionó anteriormente, se ajustaron los tiempos de maduración para ello se añadieron dos días en compensación del tiempo previo a la congelación. Por lo tanto, los tiempos de maduración se referirán como 2, 9, 16 o 23 días.

\section{Determinación de la fuerza de corte (FC)}

La FC se determinó según los lineamientos de la Asociación Americana de Ciencia de la Carne (AMSA, 1995). Se introdujo un termopar de hierro-constatán [SB1] (Omega Engineering Inc., Stamford, USA) en el centro geométrico de cada corte. Todas las porciones se cocinaron en grupos de dos, en una parrilla eléctrica (modelo DEG-22 1500 W, Daewoo Electronics México, México) a una temperatura interna de $70^{\circ} \mathrm{C}$, controlada con un termómetro portátil.

Los cortes se enfriaron a temperatura ambiente hasta alcanzar $20-25{ }^{\circ} \mathrm{C}$ y después se obtuvieron de cada corte y de manera paralela a la fibra muscular, 6-8 cilindros de $1.27 \mathrm{~cm}$ de diámetro con un aparato automático. Al final, los cilindros se cortaron de forma perpendicular a la fibra muscular en la máquina de Warner-Bratzler, donde se utilizó la cuchilla de G-R Manufacturing (Manhattan, KS, USA). Se registró y promedió la fuerza de corte máxima de cada cilindro para obtener un valor de FC único por muestra.

\section{Análisis estadístico}

Los datos se analizaron con el procedimiento de "Modelos Lineales Generalizados del programa Statgraphics Centurion XV (StatPoint, Inc., 1982-2007). El modelo empleado incluyó los efectos fijos del músculo, el tiempo de maduración y la interacción de ambos. Los datos se examinaron por fenotipo. Cuando se obtuvieron valores $\mathrm{F}$ significativos, las medias se discriminaron por medio de la prueba de rangos múltiples de Tukey. En caso de interacción significativa, se usó una "prueba $\mathrm{t}^{\prime \prime}$ para contrastar las medias.

\section{Resultados y discusión}

La interacción músculo y tiempo de maduración influyó significativamente en la FC de la carne de los toretes Charolais $(P=0.0008)$ y Brahman $(P<0.0001)$. Por lo tanto, si se observa la FC de músculos individuales a lo largo del tiempo de maduración, se obtendrá información más precisa e ilustrativa. El efecto de la combinación de la congelación, descongelación y maduración disminuyó significativamente $(\mathrm{P}<0.05)$ la FC de los músculos de ambos fenotipos, excepto la de biceps brachii, brachialis, y supraspinatus (Cuadro 1). Estos resultados coinciden con reportes previos que mencionan el efecto limitado de la maduración sobre la FC del brachialis 
Cuadro 1. Medias de mínimos cuadrados de la fuerza de corte $(\mathrm{N})$ para los músculos del cuarto delantero en toretes de Brahman y Charolais, congelados por una semana, descongelados por $24-30$ horas a $2-4^{\circ} \mathrm{C}$ y madurados durante diferentes periodos

\begin{tabular}{|c|c|c|c|c|c|}
\hline \multirow{2}{*}{ Músculo } & \multicolumn{4}{|c|}{ Tiempo de maduración, días } & \multirow{2}{*}{ SE \pm} \\
\hline & 2 & 9 & 16 & 23 & \\
\hline \multicolumn{6}{|c|}{ Experimento 1: Brahman } \\
\hline Biceps brachii & 52.3 & 45.2 & 46.0 & 41.4 & 1.9 \\
\hline Brachialis & 56.0 & 60.2 & 52.2 & 56.2 & 1.6 \\
\hline Complexus & $62.2^{\mathrm{a}}$ & $44.8^{b}$ & $46.1^{b}$ & $36.4^{\mathrm{C}}$ & $1.3^{* * *}$ \\
\hline Splenius & $62.4^{\mathrm{a}}$ & $39.1^{b}$ & $39.6^{b}$ & $38.6^{\mathrm{b}}$ & $1.2^{* * *}$ \\
\hline Infraspinatus & $41.2^{\mathrm{a}}$ & $30.1^{b}$ & $33.4^{b}$ & $29.2^{b}$ & $0.9^{* * *}$ \\
\hline Teres major & $48.9^{a}$ & $37.1^{b}$ & $41.1^{b}$ & $41.3^{b}$ & $1.0^{* * *}$ \\
\hline Rhomboideus & $49.0^{\mathrm{a}}$ & $35.4^{b}$ & $34.0^{b}$ & $39.3^{b}$ & $1.2^{* * *}$ \\
\hline Subscapularis & $51.7^{\mathrm{a}}$ & $39.4^{b}$ & $37.3^{b}$ & $39.2^{b}$ & $1.4^{* * *}$ \\
\hline Supraspinatus & 53.3 & 51.2 & 47.2 & 47.8 & 2.9 \\
\hline Triceps brachii (long head) & $53.0^{\mathrm{a}}$ & $37.8^{b}$ & $39.6^{b}$ & $40.5^{b}$ & $1.1^{* * *}$ \\
\hline Triceps brachii (lateral head) & $63.1^{\mathrm{a}}$ & $56.2^{b}$ & $53.4^{b}$ & $50.2^{b}$ & $1.3^{* * *}$ \\
\hline \multicolumn{6}{|c|}{ Experimento 2: Charolais } \\
\hline Biceps brachii & 50.0 & 44.0 & 45.0 & 45.9 & 3.6 \\
\hline Brachialis & 58.2 & 51.1 & 59.3 & 57.3 & 1.3 \\
\hline Complexus & $46.3^{a}$ & $32.8^{b}$ & $34.6^{b}$ & $36.9^{b}$ & $1.0^{* * *}$ \\
\hline Splenius & $45.3^{a}$ & $34.8^{b}$ & $32.9^{b}$ & $33.5^{b}$ & $0.8^{* * *}$ \\
\hline Infraspinatus & $34.4^{\mathrm{a}}$ & $29.8^{b}$ & $27.2^{b}$ & $27.4^{b}$ & $0.7^{* * *}$ \\
\hline Teres major & $46.9^{a}$ & $38.1^{b}$ & $38.0^{b}$ & $38.4^{b}$ & $2.4^{* * *}$ \\
\hline Rhomboideus & $57.7^{a}$ & $31.9^{b}$ & $33.7^{b}$ & $36.5^{b}$ & $1.3^{* * *}$ \\
\hline Subscapularis & $42.0^{\mathrm{a}}$ & $32.6^{b}$ & $29.7^{b}$ & $29.4^{b}$ & $1.0^{* * *}$ \\
\hline Supraspinatus & 48.3 & 43.0 & 42.2 & 43.9 & 1.8 \\
\hline Triceps brachii (long head) & $51.6^{a}$ & $37.1^{b}$ & $39.0^{b}$ & $37.2^{b}$ & $0.8^{* * *}$ \\
\hline Triceps brachii (lateral head) & $57.1^{\mathrm{a}}$ & $58.5^{a}$ & $51.0^{\mathrm{ab}}$ & $48.4^{b}$ & $1.4^{*}$ \\
\hline
\end{tabular}

a, b Medias con diferente superíndice en la misma fila son diferentes significativamente $(P<0.05)$

* $\quad \mathrm{P}<0.05 ;{ }^{* * *} \mathrm{P}<0.001$

(Belew et al., 2003; Von Seggern et al., 2005) y del supraspinatus (Hildrum et al., 2009; Rhee et al., 2004). Por el contrario, los resultados de este trabajo no coinciden con otros estudios que clasificaron al biceps brachii de canales USDA Choice y Select como suave (Belew et al., 2003; Von Seggern et al., 2005). No obstante, algunos músculos de machos jóvenes sin castrar a menudo tienen una respuesta a la maduración mucho más pobre que la de hembras o machos castrados (Claus et al., 2010; Mach et al., 2009; Rubio et al., 2007). La FC del triceps brachii (cabeza lateral), mientras maduraba se mantuvo alta; resultado similar al obtenido con machos castrados y hembras (Gruber et al., 2006; Stelzleni et al., 2007; Von Seggern et al., 2005), y con machos enteros (Simões et al., 2005).

Por otra parte, llama la atención que en estas observaciones, el ablandamiento de los músculos (excepto el complexus, de Brahman) ocurrió hasta los 
9 días. Después de este tiempo no se observó disminución significativa en la FC. Lo anterior es difícil de explicar puesto que no hay consenso sobre lo que sería el tiempo adecuado para madurar este tipo de carne. Investigaciones como las de Bratcher et al., 2005; Gruber et al., 2006; King et al., 2009; Nelson et al., 2004; Von Seggern et al., 2005 recomiendan tiempos de maduración que varían entre 5 y 21 días, según el grado de calidad de la canal y del músculo en particular). Sin embargo, la mayoría de estos estudios fueron realizados con machos castrados y hembras, mientras que el análisis con toretes, que por lo regular no entran en los sistemas de clasificación de canales, son muy escasos. Por otro lado, los estudios de maduración siguen una metodología en la que la congelación de la carne se hace después del periodo de maduración. Bajo este esquema, la carne de machos sin castrar ha mantenido altos valores de FC, aún después de una larga maduración (Hildrum et al., 2009; Li et al., 2012; Vieira et al., 2009). Sin embargo, se ha observado que la congelación de la carne antes de la maduración, reduce drásticamente la actividad de la calpastatina (Koohmaraie, 1990; Crouse y Koohmaraie, 1990). Como consecuencia de esto, la estrategia usada aquí, que incluye la congelación y descongelación antes de la maduración puede llegar a acelerar el proceso de ablandamiento. Por lo tanto, es posible que los músculos seleccionados hayan alcanzado la mínima FC posible a los 9 días de maduración. Este razonamiento se fundamenta, además, en los recientes hallazgos del estudio de Grayson et al. (2014) en el que se observó que la carne congelada, descongelada y madurada por 14 días fue más suave que la que se maduró por 28 días. De esta forma, la estrategia congelación-descongelación-maduración parece ser una alternativa viable para contrarrestar la resistencia a la maduración, que han mostrado los músculos del cuarto delantero de machos enteros.

La mayoría de los músculos de ambos genotipos tuvieron medias de FC a los 9 días por debajo de lo reportado con machos enteros. Por ejemplo, Vieira et al. (2009) observó valores de FC superiores a $46 \mathrm{~N}$ en el músculo longissimus thoracis madurado por 10 días y congelado por 90 días antes de medir la FC. Por su parte, Li et al. (2012) también encontraron altos valores de FC (> $60 \mathrm{~N}$ ) en el músculo longissimus lumborum de bovino Chino Amarillo, bajo diferentes regímenes de enfriamiento y maduración hasta 21 días. Hildrum et al. (2009) en sus observaciones con machos enteros de la raza Norwegian Red, reportó valores de FC arriba de $50 \mathrm{~N}$ para el músculo triceps brachii [cabeza larga] madurado por 9 días (sin haberse congelado). Esto es casi $15 \mathrm{~N}$ mayor que la media de FC a los 9 días que se obtuvo para el mismo músculo en esta valoración. Asimismo, la FC reportada por Hildrum et al. (2009) para el infraspinatus sometido a 9 días de maduración es cerca de $10 \mathrm{~N}$ mayor que la observada en este trabajo. Además, resulta interesante apuntar que los músculos teres major, rhomboideus, y triceps brachii [cabeza larga] tuvieron un promedio de FC a los 9 días, igual o menor que la reportada para carne de novillos madurada por 14 días (Bratcher et al., 2005; Rhee et al., 2004; Stelzleni et al., 2007).

Conviene señalar que, es difícil realizar comparaciones consistentes entre los valores específicos de FC de las diversas investigaciones por el empleo de metodologías distintas. Sin embargo, 4 músculos de toretes Brahman y 7 de Charolais alcanzaron valores de $\mathrm{FC}<38.22 \mathrm{~N}$, lo cual es típico de carne de res suave (Belew et al., 2003). Por otra parte, aunque los animales fueron sacrificados en distintos rastros, los valores obtenidos apoyan las observaciones de que la carne 
de animales $B$. taurus es más suave que la proveniente de animales $B$. indicus (Bidner et al., 2002; Bonilha et al., 2008; Shackelford et al., 1995). Se confirma la suavidad superior del infraspinatus, ampliamente documentada (Bratcher et al., 2005; Gruber et al., 2006; Rhee et al., 2004; Searls et al., 2005; Stelzleni et al., 2007); así como el potencial para agregar valor a otros músculos del cuarto delantero. Nuestros resultados también respaldan lo sugerido por Bratcher et al. (2005) acerca de que la base de la fabricación y comercialización de la carne debería ser por músculo.

Estos hallazgos muestran que algunos de los músculos del cuarto delantero de toretes pueden producir carne suave si se congelan antes de madurarse, aún cuando provengan de animales tratados con zilpaterol. Así, congelar y descongelar la carne antes de madurarla serviría para mejorar la suavidad de la carne de machos jóvenes sin castrar. Se considera que este descubrimiento es de gran importancia para la ganadería bovina de carne en México, donde los toretes representan más del 80\% de la población de sacrificio (Mendez et al., 2009) y el zilpaterol es ampliamente utilizado en la engorda del ganado (Camponova, 2006).

Aunque es poco probable que esta estrategia sea usada de manera general por la industria cárnica en el futuro, puede ser una alternativa para mercados específicos. Particularmente, cuando se maneje carne dura que necesite largos periodos de maduración para ablandarse. En la muestra estudiada, algunos músculos del cuarto delantero de toretes alcanzaron valores de FC típicos de carne suave, después de ser congelados por una semana y madurados por 9 días. Se necesita más investigación para evaluar los efectos de la congelación sobre las características sensoriales de este tipo de carne, además de otros atributos de calidad de importancia económica (jugosidad, textura, color, etc.).

\section{Conclusiones}

Los resultados muestran que la estrategia congelación-descongelación-maduración de los músculos del cuarto delantero de toretes estudiados en este trabajo, mejora significativamente la suavidad de la carne. Se espera que análisis futuros permitan optimizar el proceso de ablandamiento y evaluar el impacto potencial en otros atributos de calidad de la carne.

\section{Financiamiento}

La Unidad de Apoyo a la Investigación en Escuelas y Facultades (UAIFE) de la Universidad Nacional Autónoma de México (UNAM) financió este estudio.

\section{Agradecimientos}

Agradecemos a Carolyn Brown, Kendra Shannon y Sylvia Welke de UNAM-Canadá por su apoyo en la corrección de estilo del manuscrito en idioma inglés. 


\section{Conflictos de interés}

Los autores estipulan que no tienen conflictos de interés.

\section{Contribución de los autores}

María Salud Rubio Lozano: diseñó, consiguió financiamiento y dirigió el proyecto, revisó la información y dirigió el manuscrito y las revisiones.

Esmeralda Vanessa Pérez Buitrón: fue la estudiante que preparó su tesis de maestría dentro de este proyecto. Ella hizo los experimentos, analizó los datos y escribió su tesis en español, además de revisar la versión inglesa del mismo.

Rubén Danilo Méndez Medina: colaboró en el diseño del proyecto, en el análisis de los resultados y en la revisión del manuscrito.

Adrián Chávez Gómez: participó en la conducción de los experimentos, en el análisis de datos y en la revisión del manuscrito.

Enrique Jesús Delgado Suárez: contribuyó con el diseño del proyecto y ayudó con el análisis estadístico de los datos. También preparó el manuscrito en inglés.

\section{Referencias}

1) AMSA. 1995. Research guidelines for cookery, sensory evaluation, and instrumental tenderness measurements of fresh meat. In: Chicago, IL: American Meat Science Association and National Livestock and Meat Board.

2) Belew, J.B., Brooks, J.C., McKenna, D.R., and Savell, J.W. 2003. Warner-Bratzler shear evaluations of 40 bovine muscles. Meat Science. 64(4): 507-512.

3) Bidner, T.D., Wyatt, W.E., Humes, P.E., Franke, D.E. and Blouin, D.C. 2002. Influence of Brahman-derivative breeds and Angus on carcass traits, physical composition, and palatability. Journal of Animal Science. 80: 2126-2133.

4) Bonilha, S.F.M., Tedeschi, L.O., Packer, I.U., Razook, A.G., Alleoni, G.F., Nardon, R.F. and Resende, F.D. 2008. Evaluation of carcass characteristics of Bos indiCus and tropically adapted Bos taurus breeds selected for postweaning weight. Journal of Animal Science. 86(8): 1770-1780.

5) Bratcher, C.L., Johnson, D.D., Littell, R.C. and Gwartney, B.L. 2005. The effects of quality grade, aging, and location within muscle on Warner-Bratzler shear force in beef muscles of locomotion. Meat Science. 70(2): 279-284.

6) Camponova. 2006. Suministran anabólicos al 70 por ciento del ganado en México. 2000Agro Revista Industrial del Campo. Nov 9. Sección Agroindustria. http://www.2000agro.com.mx/agroindustria/suministran-anabolicos-a-70-porciento-del-ganado-en-mexico/.

7) Claus, H.L., Dikeman, M.E., Murray, L., Brooks, J.C., Shook, J., Hilton, G.G., Lawrence, T.E., Mehaffey, J.M., Johnson, B.J., Allen, D.M., Streeter, M.N., Nichols, W.T., Hutcheson, J.P., Yates, D.A., Miller, M.F., Hunt, M.C. and Killefer, J. 2010. Effects of supplementing feedlot steers and heifers with zilpaterol hydrochloride on Warner-Bratzler shear force interrelationships of steer and heifer longissimus lumborum and heifer triceps brachii and gluteus medius muscles aged for 7, 14 and 21d. Meat Science 85(2): 347-355.

8) Crouse, J.D. and Koohmaraie, M. 1990. Effect of freezing of beef on subsequent postmortem aging and shear force. Journal of Food Science. 55(2): 573-574.

9) Chávez, A., Pérez, E., Rubio, M.S., Méndez, R.D., Delgado, E.J. and Díaz, D. 2012. Chemical composition and cooking properties of beef forequarter muscles of Mexican cattle from different genotypes. Meat Science. 91(2): 160-164. 
10) Grayson, A.L., King, D.A., Shackelford, S.D., Koohmaraie, M. and Wheeler, T.L. 2014. Freezing and thawing or freezing, thawing, and aging effects on beef tenderness. Journal of Animal Science. 92: 2735-2740.

11) Gruber, S.L., Tatum, J.D., Scanga, J.A., Chapman, P.L., Smith, G.C. and Belk, K.E. 2006. Effects of postmortem aging and USDA quality grade on Warner-Bratzler shear force values of seventeen individual beef muscles. Journal of Animal Science. 84(12): 3387-3396.

12) Hildrum, K.I., Rodbotten, R., Hoy, M., Berg, J., Narum, B. and Wold, J.P. 2009. Classification of different bovine muscles according to sensory characteristics and Warner Bratzler shear force. Meat Science. 83(2): 302-307.

13) King, D.A., Wheeler, T.L., Shackelford, S.D. and Koohmaraie, M. 2009. Comparison of palatability characteristics of beef gluteus medius and triceps brachii muscles. Journal of Animal Science. 87(1): 275-284.

14) Koohmaraie, M. 1990. Quantification of Ca2(+)-dependent protease activities by hydrophobic and ion-exchange chromatography. Journal of Animal Science. 68: 659-665.

15) Li, K., Zhang, Y., Mao, Y., Cornforth, D., Dong, P., Wang, R., Zhu, H. and Luo, X. 2012. Effect of very fast chilling and aging time on ultra-structure and meat quality characteristics of chinese yellow cattle M. Longissimus lumborum. Meat Sci. 92(4),: 795-804.

16) Mach, N., Bach, A., Realini, C.E., Font i Furnols, M., Velarde, A. and Devant, M. 2009. Burdizzo pre-pubertal castration effects on performance, behaviour, carcass characteristics, and meat quality of holstein bulls fed high-concentrate diets. Meat Science. 81(2): 329-334.

17) Mendez, R.D., Meza, C.O., Berruecos, J.M., Garces, P., Delgado, E.J. and Rubio, M.S. 2009. A survey of beef carcass quality and quantity attributes in Mexico. Journal of Animal Science. 87(11): 3782-3790.

18) Molina, M.E., Johnson, D.D., West, R.L. and Gwartney, B.L. 2005. Enhancing palatability traits in beef chuck muscles. Meat Science. 71(1): 52-61.

19) Nelson, J.L., Dolezal, H.G., Ray, F.K. and Morgan, J.B. 2004. Characterization of certified Angus beef steaks from the round, loin, and chuck. Journal of Animal Science. 82: 1437-1444.

20) Rhee, M. S., Wheeler, T.L., Shackelford, S.D. and Koohmaraie, M. 2004. Variation in palatability and biochemical traits within and among eleven beef muscles. Journal of Animal Science. 82: 534-550.

21) Rubio L.M.S., Méndez M.R.D. and Huerta-Leidenz, N. 2007. Characterization of beef semimembranosus and adductor muscles from US and Mexican origin. Meat Science. 76(3): 438-443.

22) Searls, G.A., Maddock, R.J. and Wulf, D.M. 2005. Intramuscular tenderness variation within four muscles of the beef chuck. Journal of Animal Science. 83: 2835-2842.

23) Shackelford, S.D., Wheeler, T.L. and Koohmaraie, M. 1995. Relationship between shear force and trained sensory panel tenderness ratings of 10 major muscles from Bos indicus and Bos taurus cattle. Journal of Animal Science. 73: 3333-3340.

24) Simões, J.A., Mendes, M.I. and Lemos, J.P.C. 2005. Selection of muscles as indicators of tenderness after seven days of ageing. Meat Science. 69(4): 617-620.

25) Stelzleni, A.M., Patten, L.E., Johnson, D.D., Calkins, C.R. and Gwartney, B.L. 2007. Benchmarking carcass characteristics and muscles from commercially identified 
beef and dairy cull cow carcasses for Warner-Bratzler shear force and sensory attributes. Journal of Animal Science. 85(10): 2631-2638.

26) Vieira, C., Diaz, M.T., Martinez, B. and Garcia-Cachan, M.D. 2009. Effect of frozen storage conditions (temperature and length of storage) on microbiological and sensory quality of rustic crossbred beef at different states of ageing. Meat Sci. 83(3): 398-404.

27) Von Seggern, D.D., Calkins, C.R., Johnson, D.D., Brickler, J.E. and Gwartney, B.L. 2005. Muscle profiling: Characterizing the muscles of the beef chuck and round. Meat Science. 71(1): 39-51.

28) Yadata, M.A., Werner, C., Tibbo, M., Wollny, C.B.A. and Wicke, M. 2009. Assessment of the sensory quality and shelf stability of selected Horro beef muscles in Ethiopia. Meat Science. 83(1): 113-119 